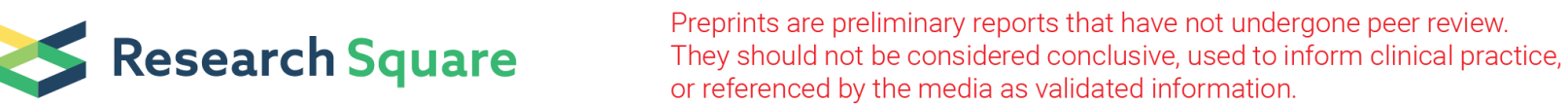

\section{Increased levels of serum Hcy and UA as well as the thickness of carotid intima-media correlates with the severity of coronary artery lesions}

\section{Changming Tan}

The Second Xiangya Hospital of Central South University

Jianming Li ( $D$ lijianming19nm@163.com)

The Second Xiangya Hospital of Central South University https://orcid.org/0000-0003-2582-4666

Yangzhao Zhou

The Second Xiangya Hospital of Central South University

\section{Feng Xu}

Central South University

Research article

Keywords: coronary heart disease, homocysteine, serum uric acid, carotid intima-media thickness, correlation

Posted Date: August 31st, 2019

DOI: https://doi.org/10.21203/rs.2.13693/v1

License: (9) This work is licensed under a Creative Commons Attribution 4.0 International License. Read Full License 


\section{Abstract}

Background To investigate the correlation between serum homocysteine (Hcy) and uric acid (UA) levels as well as carotid intima-media thickness (IMT) and the severity of coronary artery lesions in elderly patients with coronary heart disease (CHD). Methods A total of 156 elderly patients with CHD in the experimental group were further divided into low, middle and high score groups. Serum Hcy and UA levels, IMT, Gensini and Sullivan scores were measured and analyzed by Pearson correlation. The risk factors for $\mathrm{CHD}$ and the correlation were analyzed by single factor analysis and logistic regression. Results The serum levels of Hcy, UA and the IMT in the experimental group were significantly higher than those in the control group (all P < 0.001). Gensini scores showed that the levels of serum Hcy, UA, and the IMT in middle and high score groups were significantly higher than those in the low score group, while the high score group had the significant highest level (all $P<0.001$ ). Serum Hcy, UA levels and the IMT were positively correlated with Gensini and Sullivan scores (all $P<0.001$ ). And male gender, smoking, history of hypertension, systolic pressure, pulse pressure, Hcy, UA, IMT, and BMI were independently correlated with the severity of CHD (all P < 0.05).Conclusions The serum levels of Hcy and UA and the IMT in patients with CHD were significantly increased, and were positively correlated with the severity of coronary artery lesions, which may serve as diagnostic indicators.

\section{Background}

Coronary heart disease (CHD) is a common disease of circulatory system. It is caused by many factors including the person's living environment, dietary habits, heredity and so on. Coronary atherosclerotic heart disease, the most common type of CHD, is caused by coronary atherosclerotic stenosis, which leads to necrosis of the myocardium due to the insufficient blood supply. In addition, broadly speaking, the stenosis of the lumen caused by extensive inflammation and embolism also belong to CHD. For a long time, the "gold standard" for evaluating the severity of coronary artery lesions has been the coronary angiography, but it is too traumatic to be suitable for elderly patients. However, most CHD patients are middle-aged and elderly. Therefore, it is important to identify and develop appropriate non-invasive tests and indicators for clinical evaluation of the severity of CHD.

In recent years, it has been shown that the severity of CHD coronary artery lesions is related to the levels of serum homocysteine (Hcy) and uric acid (UA) and the carotid intima-media thickness (IMT) [1]. Hcy is a sulfur-containing amino acid produced during methionine metabolism and is significantly associated with the severity of coronary artery lesions. It plays an important role in the development of cardiovascular diseases such as atherosclerosis, aneurysm and myocardial infarction [2]. Hcy can promote hypertrophy and proliferation of neutrophils, which can directly damage vascular endothelial cells and their function, thereby, increasing the risk of cardiovascular disease. As a specific component of the cardiovascular structure, UA is one of the most important water-soluble endogenous antioxidants in the human body [3]. UA can aggravate atherosclerosis by promoting renal tubule reabsorption of sodium ions. Some studies have shown that UA is an independent risk factor and an independent predictor of coronary atherosclerosis [4]. It's also suggested that UA has a protective effect due to its free radical 
scavenging effect which is 10 times better than that of other anti-oxidants [5]. It can chelate metal ions and inhibit the Fenton reaction. It can, hence, prevent cardiovascular damage by oxidative stress and thus play a protective role. It is considered that the lowering UA level can improve the prognosis of CHD patients with acute myocardial infarction. A study found that when coronary atherosclerosis occurs, the IMT increases [6]. IMT can be easily and noninvasively measured to observe atherosclerosis in patients with $\mathrm{CHD}$ and is a good indicator of the severity of coronary artery lesions. The most important characteristics of $\mathrm{CHD}$ are irreversibility, sudden death rate and a slow disease progression. Therefore, early diagnosis and treatment of CHD is of great importance. What's more, the diagnosis of the severity of CHD mainly depends on the assessment of the severity of coronary artery lesions [7]. This diagnosis can help in appropriate treatment and prognosis of CHD. Hence, in the present study, we have explored the correlation between serum levels of Hcy and UA as well as the IMT, and the severity of coronary artery lesions in elderly patients with CHD.

\section{Methods}

\section{General data}

This study was approved by the Medical Ethics Committee of The Second Xiangya Hospital of Central South University. A total of 156 elderly patients admitted in our hospital for CHD diagnosed by coronary angiography from September 2017 to August 2018 were enrolled as the experimental group for retrospective analysis. According to their Gensini scores, the experimental group was further sub-divided into three groups: low score ( $\leq 20$ points, 72 cases), middle score ( $21-40$ points, 56 cases) and high score ( $\geq 40$ points, 28 cases) groups [8]. Another 54 healthy controls with normal coronary artery were enrolled as the control group. All patients and their families agreed to participate in the study and signed the informed consent document.

Inclusion criteria: All patients: (1) met the diagnostic criteria of CHD according to the Guidelines for Coronary Heart Disease, and were diagnosed by coronary angiography [9]; (2) were aged from 61 to 88 years; (3) had no history of an operation, hormone use, use of immunosuppressive agents and antibiotics in the past six months; (4) had no history of gout and hyperuricemia.

Exclusion criteria: The following patients were excluded: (1) those with serious diseases of liver, kidney, brain, lung and other basic organs; (2) those with acute and chronic infections; (3) those with malignant tumors, tuberculosis and aneurysms; (4) those with diseases of blood, endocrine and immune systems; and (5) those with lactation and pregnancy.

\section{Sample collection}

Following enrollment, $5 \mathrm{~mL}$ of fasting venous blood was collected from all study participants and after $30 \mathrm{~min}$, centrifuged at a speed of 3,000 rpm for 10 minutes. The serum was used to determine the Hcy and UA levels in the blood. The serum Hcy level was measured using circulating enzyme method with Hcy 
detector (Shenzhen Ausa Pharma Co., Ltd.) according to the kit instructions (Shanghai Jimian Industrial Co., Ltd.). UA enzyme colorimetry was used to measure serum UA level by automatic biochemical analyzer according to kit instructions (Shanghai Xinfan Biotechnology Co., Ltd). VIVIVID7 ultrasound diagnostic device (Shenzhen Kaili Biomedical Science and Technology Co., Ltd.) was used to examine the carotid arteries in the subjects in the supine position with the probe frequency ranging from $7.5 \mathrm{~Hz}$ to 12.0 $\mathrm{Hz}$.

\section{Observation parameters}

Main parameters: (1)The levels of Hcy, UA and IMT: The levels of Hcy, UA and IMT were compared between the experimental group and control group as well as among low, middle and high score group. IMT was measured as the mean of thickness between the anterior and posterior walls of the carotid arteries, including bilateral carotid bifurcation, bilateral internal carotid artery and the bilateral of the common carotid artery. It was evaluated that $0.9 \mathrm{~mm} \leq \mathrm{IMT}<1.3 \mathrm{~mm}$ was considered as thickening while IMT $\geq 1.3 \mathrm{~mm}$ was considered as atheromatous plaque formation. (2) Gensini score: All experimental group patients underwent coronary angiography by Judkins method in the catheter room of our hospital. According to the segmentation evaluation standard for the coronary artery image score of the American Heart Association, the integral for each lesion equaled the stenosis score multiplied by the lesion site integral. Each patient's score was the sum of the integral for each lesion (Gensini score) [9]. The higher the score was, the greater the degree of coronary artery stenosis was.

Secondary parameters: (1) Sullivan score: The proportion of coronary intima atherosclerotic plaque was evaluated by Sullivan score, which was positively correlated with Sullivan score. (2) The general data of experimental group and control group were included in the single factor analysis, including gender, body mass index (BMI), history of smoking, alcohol consumption, hypertension, diabetes and cardiovascular diseases, systolic, diastolic and pulse pressure, Hcy, UA and IMT. (3) The correlation of multiple factors and severity of coronary artery lesions.

\section{Statistical methods}

All data in this study were analyzed by SPSS21.0 statistical software. The level of each observation index was expressed as the mean \pm standard deviation ( \pm sd) and analyzed using F-test. All count data were expressed as percentages $(n, \%)$ and analyzed using a $\chi^{2}$ test. Pearson linear correlation analysis was used to analyze the correlation between serum Hcy, UA level as well as IMT and Gensini score, Sullivan score. The general data of experimental group and control group were included in the single factor analysis. Logistic regression analysis was used to analyze the correlation of multiple factors and severity of coronary artery lesions. The integral of coronary stenosis was taken as the dependent variable while Hcy, UA and IMT were taken as independent variables. Differences were considered significant when $\mathrm{P}<$ 0.05 . 


\section{Results}

\section{General data comparison among low, middle and high score groups}

There was no significant difference in the general data among the three groups $(P>0.05)$, as shown in Table 1.

\section{Comparison of Hcy and UA levels and IMT among low, middle and high score groups}

The serum Hcy, UA and the IMT in the middle and high score groups were significantly higher than those in the low score group (all $\mathrm{P}<0.001$ ), while those in the high score group were significantly higher than those in the middle score group (all $P<0.001$ ), as shown in Table 2 and Fig. 1.

\section{Correlation of serum Hcy, UA level and IMT with Gensini score}

Pearson correlation analysis showed that serum Hcy and UA levels and IMT were positively correlated with Gensini score in the experimental group $(r=0.476,0.517,0.528 ; P<0.001)$, respectively, as shown in Table 3 and Fig. 2.

\section{Correlation of serum Hcy and UA levels and IMT with Sullivan integral}

Pearson correlation analysis showed that serum Hcy and UA levels and IMT were positively correlated with Sullivan integral in the experimental group $(r=0.587,0.563,0.617 ; P<0.001)$, respectively, as shown in Table 4 and Fig. 3.

\section{Univariate analysis of general data in the experimental and control groups}

Univariate analysis of the general data showed that male gender, BMI, history of smoking, history of hypertension, systolic blood pressure, pulse pressure, Hcy, UA, and IMT were significantly correlated with CHD in the experimental group (all $\mathrm{P}<0.05$ ), as shown in Table 5.

\section{Logistic regression analysis of the correlation between multivariate factors and CHD}

Based on assignment table (Table 6), logistic regression analysis showed that male gender, smoking, history of hypertension, systolic blood pressure, pulse pressure, Hcy, UA, IMT, and BMI independently correlated with the severity of CHD in the experimental group (all $P<0.05)$. See Table 7. 


\section{Discussion}

There are many risk factors for developing CHD in the elderly, among which age is the most significant, followed by carotid plaque formation. In addition, B-complex vitamins, gender, obesity, smoking and alcohol can affect the severity of coronary artery disease by influencing the level of serum Hcy and UA [10]. In recent years, with the increasing incidence and mortality of CHD in the elderly, the improvement of therapeutic efficacy has been the heat spot. Under the circumstance of slow progression and high risk of late stage, the key to the effective management of CHD is timely and effective diagnosis. Studies have shown that the main method to assess the severity of CHD in patients is to evaluate the severity of coronary artery lesions, so it is important to find suitable non-invasive, low-cost evaluation markers [11].

Hcy is produced in the liver and other organs by methionine metabolic cycle. There are two ways, methionine and cysteine. Normally, Hcy has a lower serum level about $8 \mu \mathrm{mol}$ [12]. Hcy induces coronary artery disease by damaging proteins through oxidative stress, resulting in the decrease or inactivation of enzymes and enzyme receptors, along with the decrease in nitric oxide synthesis, and subsequently impairs endothelial function, ultimately hindering vascular expansion. Besides, Hcy is an important factor in promoting thrombosis. Under its increased expression, the blood is in a hypercoagulable state, which causes a large number of platelets to adhere and aggregate together. At the same time, Hcy induces lumen stenosis by stimulating the proliferation of vascular smooth muscle cells, and also results in an imbalance in the lipid, carbohydrate and protein metabolism, leading to the formation of lipid deposition plaques $[13,14]$. The observations in our study showed that the serum Hcy level in the CHD group was significantly higher than in the control group, indicating that the serum Hcy level was an independent risk factor for CHD. The study also showed that the level of serum Hcy in high and middle score groups was significantly higher than in low score group and that in high score group was even higher as compared to the middle score group. This indicated that the level of serum Hcy was positively correlated with Gensini score, namely, the severity of coronary lesions. The higher the level of serum Hcy, the more serious the coronary lesions. Various studies have found that there is a significant correlation between high serum Hcy level and CHD [15]. Wang et al. also found the significant correlation between serum Hcy level and severity of coronary artery disease [16]. These previous results are consistent with the observations in this study $[17,18]$.

UA is a water-soluble antioxidant distributed in the human cardiovascular system, which is normally stable in the serum. It is produced by purine metabolism by the oxidation of hypoxanthine to xanthine under the action of enzymes and finally to UA [19]. Some studies have pointed out that serum UA levels in the elderly diagnosed with CHD show significant changes, suggesting that it has a certain correlation with coronary artery disease [20]. In elderly CHD coronary artery disease, UA level increased as a result of increasingly producing but slowly clearing. Studies have shown elevated serum UA level can lead to sympathetic excitation and renal hemodynamic changes. To be specific, UA promotes the re-absorption of sodium through the proximal convoluted tubules of the glomeruli and accelerates the production of endothelin in blood, thus inducing CHD and also aggravating the severity of coronary artery disease [21]. This study showed that the serum UA levels in experimental group was significantly higher than in control 
group, and the serum UA level in high and middle score groups was significantly higher than that in the low score group and high score group had the highest serum UA level. This indicated that serum UA is an independent risk factor for $\mathrm{CHD}$ and was positively correlated with the severity of coronary artery disease, which was consistent with the above findings [22].

IMT is an important visual index for evaluating the severity of coronary artery disease. A study has shown that IMT is correlated with the severity of coronary artery disease and greater the IMT, the more serious $\mathrm{CHD}$ [23]. One of the pathological basis for developing CHD in the elderly is endothelial damage, which leads to lipid deposition under the intima of the vessel, promotes inflammation and plaque formation, and eventually thickens smooth muscle and hardens the vessel wall, leading to increased IMT [24]. In this study, IMT in the experimental group was significantly higher than that in the control group; IMT in middle and high score groups was significantly higher than that in low score group; while IMT in high score group was significantly higher than that in middle score group, indicating that IMT is significantly correlated with the severity of coronary artery disease. The results were consistent with those of the above studies [25].

Pearson correlation analysis showed that serum Hcy, UA level and IMT were positively correlated with Gensini and Sullivan score, respectively. Logistic regression analysis showed that male gender, smoking, history of hypertension, systolic blood pressure, pulse pressure, Hcy, UA, IMT and BMI were independently associated with the severity of CHD. The greatest disadvantage of this study is the small sample size.

\section{Conclusions}

In conclusion, the levels of serum Hcy and UA and IMT in patients with CHD were significantly increased, and the increase was positively correlated with the severity of coronary artery lesions. They can be used as diagnostic indices for evaluating the severity of $\mathrm{CHD}$ in clinical settings.

\section{Abbreviations}

Hcy, homocysteine; UA, uric acid; IMT, intima-media thickness; CHD, coronary heart disease; BMI, body mass index

\section{Declarations}

\section{Ethical approval and consent to participate}

This study was approved by the Medical Ethics Committee of The Second Xiangya Hospital of Central South University. All patients and their families agreed to participate in the study and signed the informed consent document.

\section{Consent for publication}




\section{Availability of data and materials}

The analysed data sets generated during the study are available from the corresponding author on reasonable request.

\section{Competing interests}

The authors declare that they have no competing interests.

\section{Funding}

Not applicable.

\section{Authors' contributions}

CMT contributed to the study concepts, study design, definition of intellectual content, literature research and data acquisition in addition to preparing and editing the manuscript. JML contributes to the manuscript review and guaranteed the integrity of the entire study. FX contributed to data and statistic analysis. CMT carried out the clinical studies. FX and YZZ did experimental studies. The final version of the manuscript has been read and approved by all authors, and each author believes that the manuscript represents honest work.

\section{Acknowledgements}

Not applicable.

\section{References}

1.Wang T, Sun ZW, Shao LQ, Xu XB, Liu Y, Qin M, Weng X, Zhang YX. Diagnostic Values of Serum Levels of Homocysteine and Uric Acid for Predicting Vascular Mild Cognitive Impairment in Patients with Cerebral Small Vessel Disease. Med Sci Monit. 2017;23:2217-25.

2.Pu Z, Xu W, Lin Y, He J, Huang M. Oxidative Stress Markers and Metal lons are Correlated With Cognitive Function in Alzheimer's Disease. Am J Alzheimers Dis Other Demen. 2017;32:353-9.

3.Qiu X, Gao F, Qiu Y, Bao J, Gu X, Long Y, Liu F, Cai M, Liu H. Association of maternal serum homocysteine concentration levels in late stage of pregnancy with preterm births: a nested case-control study. J Matern Fetal Neonatal Med. 2018;31:2673-7. 
4.Duan S, Zhang S, Li L, Ren C, Xie J. Carotid artery intima-media thickness associated with prognosis of intracranial branch atheromatous disease. Int J Neurosci. 2017;127:361-7.

5.Wang DY, Jiang HB, Zhou YM. Protective effects of folic acid combined with multivitamin B on target organ function in patients with lacunar cerebral infarction complicated with h-type hypertension. Chinese journal of clinical pharmacy. 2018;27:21-5.

6.Zhou SC. Effect of atorvastatin calcium combined with butylphthalide soft capsule on NIHSS score and serum homocysteine level in patients with acute cerebral infarction. Chinese journal of general medicine. 2018;30:42-4.

7.Yuan Dan, Chen RJ, Zhang XR. Study on correlation between blood glucose level and acute phase neurologic function in type 2 diabetes mellitus with acute cerebral infarction patients. Jilin Medical Journal. 2018;39:823-5.

8.Wang SL, Tian T, Zhou DH, Wang SM, Xue Y, Sun ZQ. Clinical observation of atorvastatin in the treatment of type 2 diabetes in the elderly. Chinese geriatric healthcare medicine. 2018;16:52-4.

9.Lin LT, Wwi Z, Wei RW, Huang FK, Song SP. Correlation research between serum hs-CRP, Hcy levels and carotid artery atherosclerosis of patients with dia-betes mellitus and atrial fibrillation. Chinese Journal of Clinical Healthcare. 2018;21:203-206.

10.Rong AG. Relationship between blood homocysteine, carotid atherosclerosis and 24-hour blood pressure variability in patients with essential hypertension. Thrombosis \& hemostasis. 2017;23:924-6.

11.Lv XH, Yu XD, Yue Y. Correlation analysis between serum homocysteine level and atherosclerosis in patients with prehypertension. China Practical Medical. 2017;12:47-8.

12.Song XJ. Effects of tongxinluo combined with ruisuvastatin on plasma Hcy, L-PGDS, and endothelin levels in patients with carotid atherosclerosis of H-type hypertension. Modern Journal of Integrated Traditional Chinese and Western Medicine. 2017;26:111-3.

13. Hu HZ, Hou P, Cao YZ. Application value of carotid ultrasound combined with serum homocysteine in the diagnosis of coronary atherosclerotic heart disease. Jilin Medical Journal. 2017;38:1245-8.

14.Fang GQ, Han L, Chen YH, Wang P, Duan JL. The correlation between homocysteine and carotid intima-media thickness as well as blood pressure variability in patients with type $\mathrm{H}$ hypertension. Hebei Medical Journal. 2017; 39:2927-9.

15.She CZ, Zhang ZS, Peng ZY, Chen Z, Yang CJ, Yang YZ, Lu L. Effects of Shuxuetong Injection on Intima-media Thickness, Serum CPR and Homocysteine Level of Patients with Transient Ischemic Attack. Evaluation and Analysis of Drug-Use in Hospitals of China. 2017; 17:1031-2. 
16.Wang SQ. Relationship between serum homocysteine level and carotid atherosclerosis in patients with vascular dementia. The Journal of Medical Theory and Practice. 2017;30:1818-9.

17.Nie Y, Feng XC, Chen BX, Qiu WF, Xia RH, Lu YT, Zhuang RT. Study of relation between elevated homocysteine and carotid atherosclerosis in elderly people. Chinese journal of the Frontiers of Medical Sciences (Electronic Version). 2017;9:96-100.

18.Chen XR, Xiao BR, Cheng H, Li H. Early Diagnosis Value of Carotid IMT, Hcy and D-D Levels in Patients with Acute Coronary Syndrome. Chinese journal of hemorheology. 2017;27:220-3.

19.Li L, Qin L, Lu QJ, Lu HL, Li PJ, Yang RL. Brachial artery endothelial function and carotid intima-media thickness of H-type hypertensive patients. Chinese Journal of General Practitioners. 2017;16:701-704.

20.Zhao XX, Bo LJ, Zhao HW, Li LH, Zhou YY, Wang HY. Descriptive study of the relationship between the subclinical carotid disease and biomarkers, carotid femoral pulse wave velocity in patients with hypertension. Clin Exp Hypertens. 2018;40:274-80.

21.Yann LG, Francois XS; Julien B. Performance Analysis of Single-Receiver Matched-Mode Localization. IEEE Journal of Oceanic Engineering. 2018;44:193-206.

22.Fu Y, Wei L, Yang YP. Effect of lishukang capsule on homocysteine in patients with chronic renal failure. Proceeding of Clinical Medicine. 2017; 26:735-8.

23.Liu YQ, Wang Y, Zhou Y, Han ZX. Application value and correlation of femoral artery ultrasound and SLRP1 and Hcy level detection in type 2 diabetes mellitus with myocardial infarction. Chinese Journal of Clinical Research. 2017;30:196-9.

24. He F. Effect of lipitor combined with biaspirin on serum Hcy level in patients with carotid atherosclerosis. World Latest Medicine Information. 2017;17:90-6.

25.Yin N, Sun XL, Wu HM, Hou GC, Gao HY. Effects of Alprostadil combined with Uremic Clearance Granule on Serum Parathyroid Hormone and Homocysteine Levels in Patients with Chronic Renal Failure and Its Clinical Efficacy. Advances in modern biomedicine. 2017;17:1945-8.

\section{Tables}

Table 1 General data comparison ( \pm sd, $n(\%)$ ) 


\begin{tabular}{|c|c|c|c|c|c|}
\hline Group & $\begin{array}{l}\text { Low score } \\
\text { group }(n=28)\end{array}$ & $\begin{array}{l}\text { Middle score } \\
\text { group }(n=28)\end{array}$ & $\begin{array}{l}\text { High score } \\
\text { group }(n=28)\end{array}$ & F & $\mathrm{P}$ \\
\hline $\begin{array}{l}\text { Gender (male/ } \\
\text { female) }\end{array}$ & $55 / 17$ & $42 / 14$ & $22 / 6$ & 0.243 & 0.912 \\
\hline $\begin{array}{l}\text { Average age } \\
\text { (year) }\end{array}$ & $71.91 \pm 13.82$ & $71.21 \pm 14.12$ & $73.32 \pm 14.11$ & 0.831 & 0.312 \\
\hline BMI $\left(\mathrm{kg} / \mathrm{m}^{2}\right)$ & $24.43 \pm 2.34$ & $24.35 \pm 2.31$ & $24.65 \pm 2.37$ & 0.217 & 0.918 \\
\hline $\begin{array}{l}\text { Type of CHD ( } n \text {, } \\
\%)\end{array}$ & & & & & \\
\hline Stable type & $30(41.67)$ & $23(41.07)$ & $11(39.29)$ & 0.047 & 0.977 \\
\hline Unstable type & 19 (26.39) & 15 (26.79) & $8(28.57)$ & 0.05 & 0.976 \\
\hline $\begin{array}{l}\text { Acute cardiac } \\
\text { infarction }\end{array}$ & $23(31.94)$ & $18(32.14)$ & $9(32.14)$ & 0.001 & 1.000 \\
\hline $\begin{array}{l}\text { Complications ( } n \text {, } \\
\%)\end{array}$ & & & & & \\
\hline Hypertension & $40(55.56)$ & $31(55.36)$ & $16(57.14)$ & 0.267 & 0.987 \\
\hline Diabetes & $29(40.28)$ & $24(42.86)$ & $11(39.29)$ & 0.128 & 0.937 \\
\hline Hyperlipidaemia & $43(59.72)$ & $34(60.71)$ & $17(60.71)$ & 0.016 & 0.992 \\
\hline
\end{tabular}

BMI, body mass index; CHD, coronary heart disease. 
Table 2 Comparison of Hcy and UA levels and IMT ( \pm sd)

\begin{tabular}{llll}
\hline Group & Low score group $(n=$ & Middle score group $(n=$ & $\begin{array}{l}\text { High score group }(n= \\
28)\end{array}$ \\
& 28) & $28)$ & $28.98 \pm 8.04^{* * *}$ \\
\hline Hcy & $11.34 \pm 6.83$ & $20.03 \pm 7.88^{* * *}, \# \# \#$ & \\
$(\mu \mathrm{mol} / \mathrm{L})$ & & $326.34 \pm 79.34^{* * *}, \# \# \#$ & $456.67 \pm 98.34^{* * *}$ \\
$\mathrm{UA}$ & $206.78 \pm 77.45$ & & \\
$(\mu \mathrm{mol} / \mathrm{L})$ & & $1.26 \pm 0.07^{* * *}, \# \# \#$ & $1.33 \pm 0.08^{* * *}$ \\
\hline $\mathrm{IMT}(\mathrm{mm})$ & $1.19 \pm 0.05$ & & \\
\hline
\end{tabular}

${ }^{* * *} \mathrm{P}<0.001$, compared with low score group; ${ }^{\# \# \#} \mathrm{P}<0.001$, compared with high score group.

Table 3 Correlation of serum Hcy, UA level and IMT with Gensini score

\begin{tabular}{llll}
\hline Group & Correlation coefficient & Statistic & $\mathrm{P}$ \\
\hline Hcy & 0.476 & 10.069 & 0.000 \\
UA & 0.517 & 10.091 & 0.000 \\
\hline IMT & 0.528 & 10.031 & 0.000 \\
\hline
\end{tabular}

Hcy, homocysteine; UA, uric acid; IMT, intima-media thickness. 
Table 4 Correlation of serum Hcy and UA levels and IMT with Sullivan score

\begin{tabular}{llll}
\hline Group & Correlation coefficient & Statistic & P \\
\hline Hcy & 0.587 & 10.031 & 0.000 \\
UA & 0.563 & 10.043 & 0.000 \\
\hline IMT & 0.617 & 10.034 & 0.000 \\
\hline
\end{tabular}

Hcy, homocysteine; UA, uric acid; IMT, intima-media thickness. 
Table 5 Univariate analysis of general data

\begin{tabular}{|c|c|c|c|c|}
\hline Group & $\begin{array}{l}\text { Control group }(n= \\
54)\end{array}$ & $\begin{array}{l}\text { Experimental group }(n= \\
150)\end{array}$ & $\mathrm{P}$ & $\chi^{2}$ \\
\hline Male $(n, \%)$ & $23(42.59)$ & $119(76.28)$ & 0.000 & 24.254 \\
\hline BMI $\left(\mathrm{kg} / \mathrm{m}^{2}\right)$ & $30.21 \pm 4.13$ & $24.32 \pm 2.12$ & 0.000 & 10.033 \\
\hline Smoking $(n, \%)$ & $33(61.11)$ & $60(38.46)$ & 0.004 & 8.340 \\
\hline Drinking $(n, \%)$ & $10(18.52)$ & $21(13.46)$ & 0.367 & 0.815 \\
\hline $\begin{array}{l}\text { Hypertension history } \\
(n, \%)\end{array}$ & $10(18.52)$ & $105(67.31)$ & 0.000 & 38.545 \\
\hline Diabetes history $(n, \%)$ & $8(14.81)$ & $16(10.26)$ & 0.364 & 0.823 \\
\hline CVD history $(n, \%)$ & $12(22.22)$ & $35(22.44)$ & 0.974 & 0.001 \\
\hline $\begin{array}{l}\text { Systolic pressure } \\
(\mathrm{mmHg})\end{array}$ & $122.21 \pm 8.32$ & $135.32 \pm 11.34$ & 0.000 & 9.033 \\
\hline $\begin{array}{l}\text { Diastolic pressure } \\
(\mathrm{mmHg})\end{array}$ & $76.34 \pm 5.43$ & $75.34 \pm 8.32$ & 0.317 & 1.005 \\
\hline Pulse pressure $(\mathrm{mmHg})$ & $45.43 \pm 10.32$ & $60.12 \pm 12.32$ & 0.000 & 8.560 \\
\hline Hcy $(\mu \mathrm{mol} / \mathrm{L})$ & $7.59 \pm 0.46$ & $21.03 \pm 7.66$ & 0.000 & 21.801 \\
\hline $\mathrm{UA}(\mu \mathrm{mol} / \mathrm{L})$ & $155.33 \pm 47.79$ & $363.35 \pm 85.31$ & 0.000 & 22.057 \\
\hline IMT (mm) & $0.81 \pm 0.05$ & $1.28 \pm 0.07$ & 0.000 & 53.317 \\
\hline
\end{tabular}

BMI, body mass index; CVD, cardiovascular disease; Hcy, homocysteine; UA, uric acid; IMT, intima-media thickness. 
Table 6 Univariate analysis of general data

\begin{tabular}{lcc}
\hline Index & \multicolumn{2}{c}{ Assignment } \\
\cline { 2 - 3 } & 0 & 1 \\
\hline Gender & Female & Male \\
Body mass index $\left(\mathrm{kg} / \mathrm{m}^{2}\right)$ & $\geq 26$ & $<26$ \\
\hline Smoking & No & Yes \\
\hline Hypertension history & No & Yes \\
\hline Systolic pressure $(\mathrm{mmHg})$ & $\leq 128$ & $>128$ \\
\hline Pulse pressure $(\mathrm{mmHg})$ & $\leq 52$ & $>52$ \\
\hline Hcy $(\mu \mathrm{mol} / \mathrm{L})$ & $\leq 11$ & $>11$ \\
\hline UA $(\mu \mathrm{mol} / \mathrm{L})$ & $\leq 190$ & $>190$ \\
\hline IMT $(\mathrm{mm})$ & $\leq 1$ & $>1$ \\
\hline
\end{tabular}

Hcy, homocysteine; UA, uric acid; IMT, intima-media thickness. 
Table 7 Logistic regression analysis of the correlation between multivariate factors and CHD

\begin{tabular}{llll}
\hline Index & OR & $95 \%$ CI & P \\
\hline Male & 10.22 & $1.23-70.32$ & 0.000 \\
Body mass index & 9.21 & $2.21-61.24$ & 0.000 \\
\hline Smoking & 10.23 & $2.21-31.22$ & 0.000 \\
\hline Hypertension history & 9.32 & $1.12-33.42$ & 0.000 \\
\hline Systolic pressure & 11.33 & $2.21-32.33$ & 0.000 \\
\hline Pulse pressure & 11.35 & $2,22-32.22$ & 0.000 \\
\hline Hcy & 10.88 & $1.38-71.43$ & 0.000 \\
\hline UA & 11.21 & $2.13-61.13$ & 0.000 \\
\hline IMT & 12.35 & $5.08-31.02$ & 0.000 \\
\hline
\end{tabular}

CHD, coronary heart disease; OR, odds ratio; CI,confidence interval; Hcy, homocysteine; UA, uric acid; IMT, intima-media thickness.

\section{Figures}
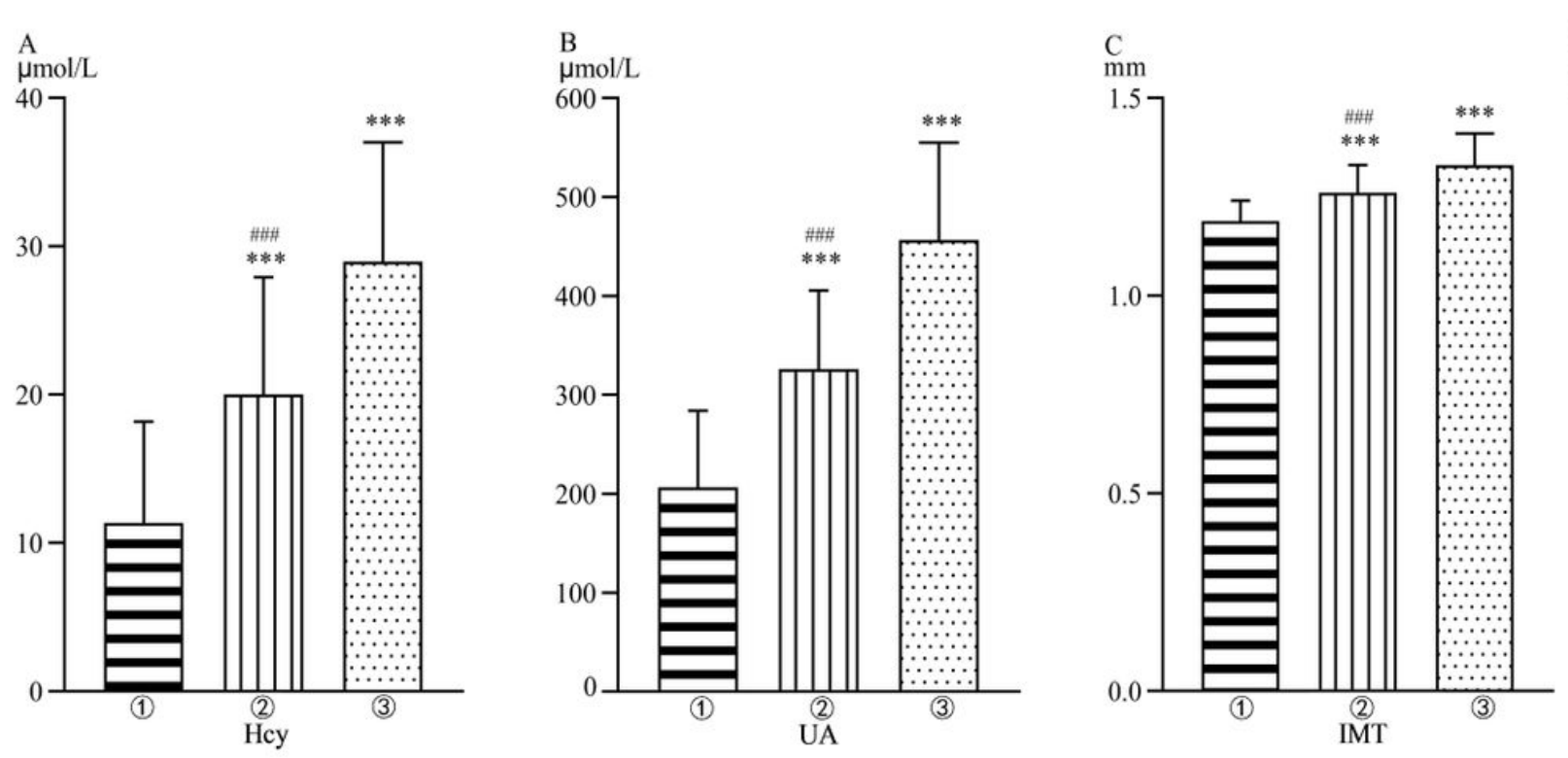

Figure 1 
Fig. 1 Comparison of Hcy(A), UA (B) and IMT (C) levels among low, middle and high groups. Compared with the low group, ${ }^{\star \star *} P<0.001$; compared with the high group, \#\#\#P<0.001. Hcy, homocysteine; $U A$, uric acid; IMT, intima-media thickness
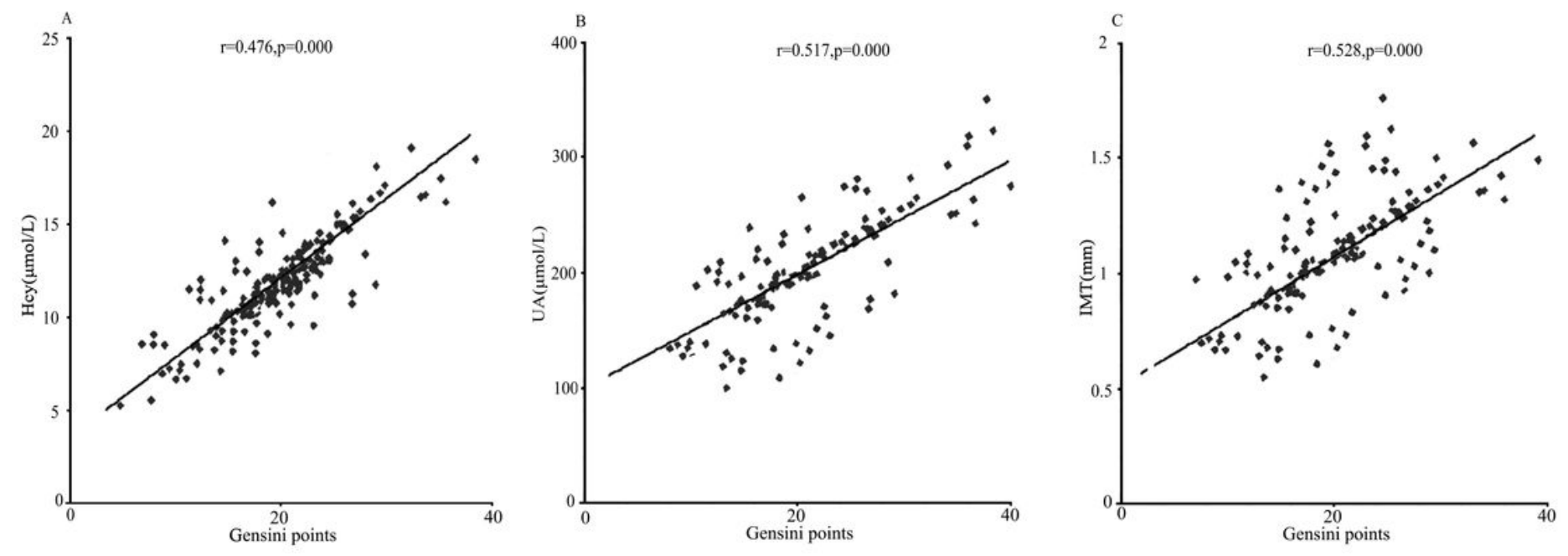

Figure 2

Fig. 2 Correlation between serum Hcy (A), UA (B) levels and IMT (C) and Gensini scores. Hcy, homocysteine; UA, uric acid; IMT, intima-media thickness
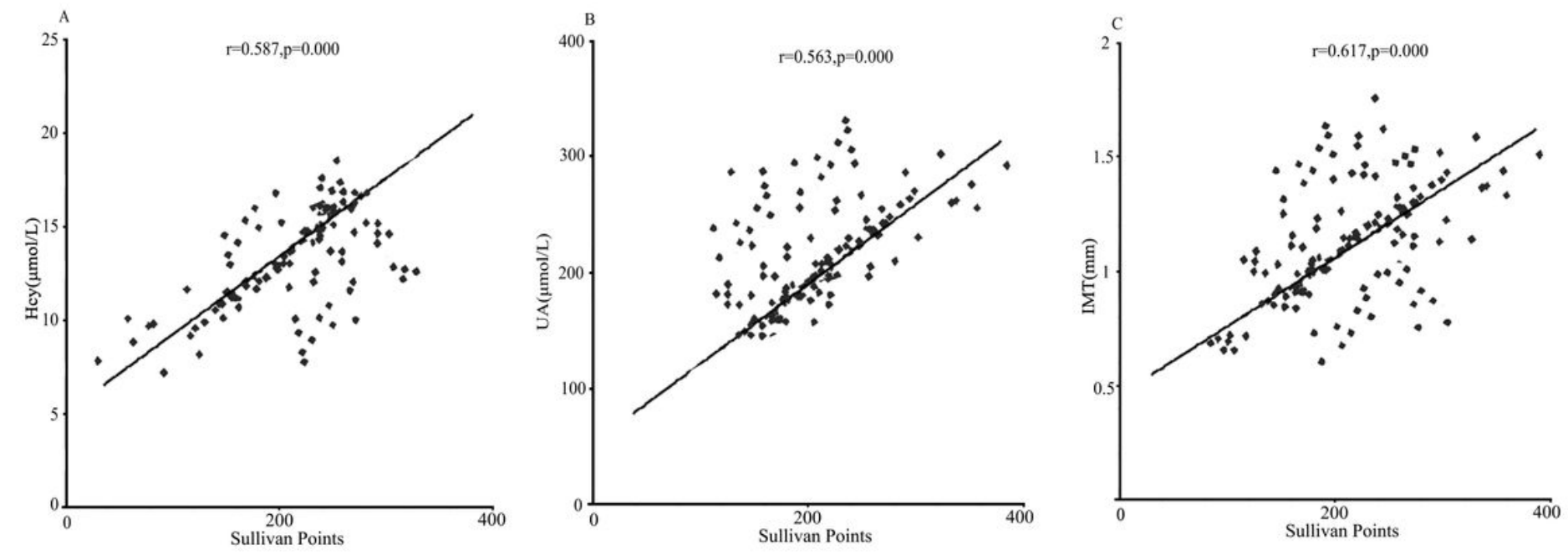

Figure 3

Fig. 3 Correlation between serum Hcy (A), UA (B) levels and IMT (C) and Sullivan scores. Hcy, homocysteine; UA, uric acid; IMT, intima-media thickness 